\title{
Parameters of a gas discharge with a liquid electrode in the process of gasification of hydrocarbon-containing waste
}

\author{
Bulat Tazmeev ${ }^{1}$, Ramilya Tazmeeva ${ }^{2}$, and Vladimir Pogosyan ${ }^{1, *}$ \\ ${ }^{1}$ Kuban State Agrarian University named after I.T. Trubilin, Krasnodar, Kalinina st., 13, Krasnodar, \\ 350044, Russian Federation \\ ${ }^{2}$ Kazan Federal University, Naberezhnye Chelny Institute, Mira st., 68/19, Naberezhnye Chelny, \\ 423810, Russian Federation
}

\begin{abstract}
A plasma generator with a liquid cathode has been developed and investigated, which makes it possible to create a plasma flow from electrolyte vapors with a temperature of up to $1800^{\circ} \mathrm{C}$ with a mass flow rate of up to $3.0 \mathrm{~g} / \mathrm{s}$ for the gasification of carbon-containing waste. An electrolyte in the form of a solution of Glauber salt in distilled water with a concentration of $0.5-1.0 \mathrm{~kg} / \mathrm{m}^{3}$ by weight was used. The current-voltage characteristics of the discharge are constructed. The optimal electrical and thermal parameters of the plasma flow are determined experimentally.
\end{abstract}

Currently, there is an acute shortage of energy resources. Deposits of hydrocarbon raw materials are depleted, and their cost periodically increases. In this regard, there is a need to search for other sources and methods of obtaining such raw materials and generating electric energy.

There are various ways to solve this problem. One of the methods is the processing of waste containing hydrocarbon compounds and the production of synthesis-gas. To do this, you can use a fully controlled system consisting of a plasma reactor and an autonomous mini-thermal power plant. Industrial waste containing hydrocarbon compounds is decomposed in the reactor. The result is a gas identical in composition to natural gas. The mini power plant converts the resulting gas into electrical energy.

The combustion of synthesis-gas generates a large amount of thermal energy, which is also a valuable resource. This energy can be used for both hot water supply and indoor heating.

Gasification of waste is undoubtedly a more efficient and technologically advanced way to process it. This method of solid waste disposal has a significant distinguishing feature from traditional incineration, which consists in the fact that a high-temperature environment is created by the electric discharge plasma. The process is easily automated using the available tools, which allows us to control and adjust the parameters with sufficient accuracy. This, in turn, makes it possible to minimize the formation of complex chemical compounds that are obtained during conventional waste incineration. Complex organic compounds decompose in the medium of steam-water plasma due to high temperature,

* Corresponding author: vpogosyan@yandex.ru 
from $1700^{\circ} \mathrm{C}$ and more. Thus, it is possible to achieve a level of high standards for the minimum emission of pollutants.

In the vast majority of cases, arc-discharge plasma torches are used to produce a plasma medium [1-4]. Gas or water vapor is blown through the discharge area. As a result, an active medium is created for decomposition of complex compounds. But the use of superheated water vapor as a plasma-forming medium creates a number of technical problems that reduce the reliability and service life of power plants. In arc plasma generators, refractory electrodes are used, which have an insufficiently long service life and a high cost. The use of water vapor leads to an even greater reduction in the duration of the effective functionality of electrodes and the rapid burnout of refractory inserts. The conductive elements of the installations are abundantly covered with water condensate. Therefore, there is a need to create a reliable protection of electrodes from the action of water vapor. In addition, an integral and important part is a powerful superheated water vapor generator, which determines the efficiency of the entire system.

Plasma generation by means of a liquid cathode discharge has a number of advantages in comparison with the electric arc method. There is no need to use an expensive and powerful steam generator. Vapor-air plasma is obtained as a result of evaporation of the electrolyte under the action of the heat flow from an electric discharge. The higher energy efficiency of the steam-water plasma is ensured by the absence of such components as nitrogen, which is present in the composition of the air plasma and does not contribute to increasing the thermal efficiency. Nitrogen is a ballast component. The possibility of formation of oxides of nitrogen, sulfur, etc. is excluded. This ensures a high ecological purity of the treatment process and a higher yield of a final product. This is facilitated by the fact that carbon raw materials in the composition of solid household waste are oxidized by superheated steam: $\mathrm{C}+\mathrm{H}_{2} \mathrm{O} \rightarrow \mathrm{H}_{2}+\mathrm{CO}$.

Plasma generators with a liquid cathode make it possible to create a plasma flow with the specified characteristics [5-7]. To create a plasma flow, a plasma torch with a liquid cathode was developed (Figure 1). The average mass temperature of the electrolyte vapor was $1800^{\circ} \mathrm{C}$, the mass flow rate was $3.0 \mathrm{~g} / \mathrm{s}$. To increase the effective heat transfer, a device has been developed for supplying additional plasma-forming gas through the discharge region in a ratio of 1:1 with the mass flow rate of the electrolyte.
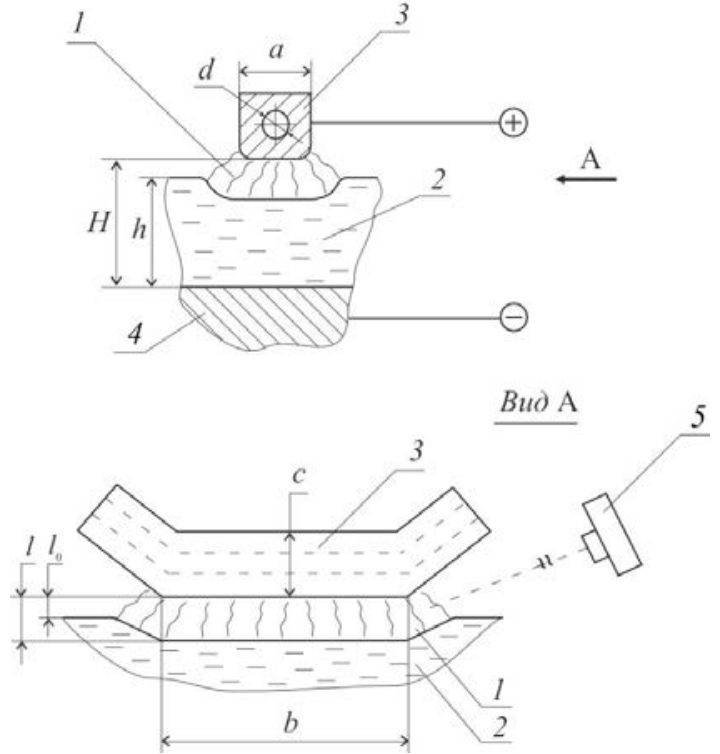

Fig. 1. Gas-discharge unit of the experimental installation. 1-Discharge region, 2-electrolyte, 3-metal anode, 4-current supply, 5-camera. 
The elements of the plasma generator power supply are quite simple and reliable in operation. A serial step-up transformer is used. The secondary windings of the transformer are connected to a three-phase two-half-period rectifier with grounding of the negative pole of the source. The electrical parameters of the discharge were controlled by gradually changing the voltage on the primary winding of the transformer. The parameters were controlled by measuring devices of the accuracy class 0.2 .

The construction of the current-voltage characteristics (Figure 2) showed that they have a growing character. This is also a preemptive factor, since in this case there is no need to use the ballast resistance. The absence of ballast resistance significantly reduces power losses and increases the thermal efficiency of the generator.

The range of changes in the main parameters: discharge current $5 \div 8 \mathrm{~A}$, generator power $6 \div 21 \mathrm{kWt}$.

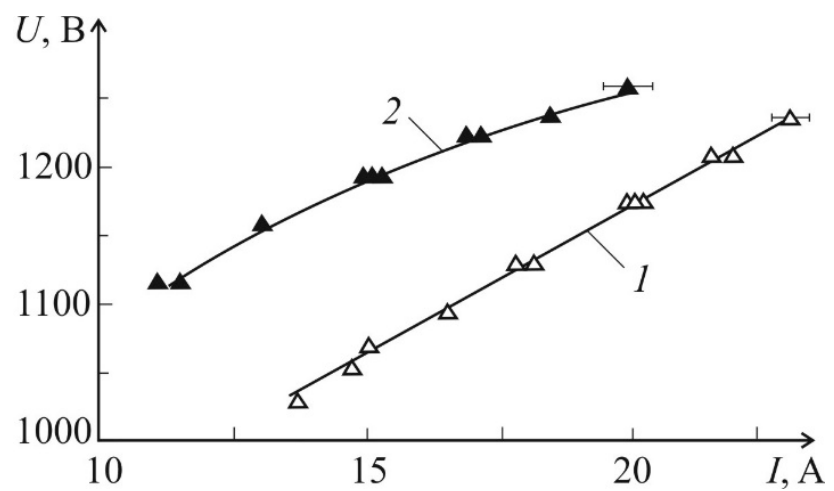

Fig. 2. Current-voltage characteristics of a gas discharge. Anode - plate. Electrolyte - water solution $\mathrm{Na}_{2} \mathrm{SO}_{4}$. Interelectrode distance: $1-l_{0}=2 \mathrm{~mm} ; 2-4$.

In many cases, the electrolyte can be tap water or industrial water with a low salt content. This is due to the low cost and practicality of using generators with liquid electrodes. In this work, a solution of Glauber's salt in distilled water is used. The concentration of $0.5-1.0 \mathrm{~kg} / \mathrm{m}^{3}$ by weight was selected. The specific electrical conductivity at this concentration and under normal conditions was $0.08-0.27 \mathrm{Cm}$. The choice of physical parameters of the electrolyte is dictated by a number of conditions: the formation of deposits on the current leads is excluded, the electric discharge must maintain a volumetric structure to maintain stable modes of plasma chemical reactions, the formation of counteragent channels in the discharge region is not allowed, the electrolyte must undergo circular recirculation including the heat exchanger while maintaining its physical properties. Experimental studies have shown that the selected electrolyte fully meets the specified conditions. Diffuse discharge combustion is maintained over a wide range of currents at atmospheric and overpressure without the phenomenon of discharge contraction. This indicates that with increasing current and pressure, there is no formation of radial inhomogeneities in the discharge region. The energy balance conditions in the current range of 10-30 A (Figure 2) remain unchanged.

The parameters of the steam-gas flow were controlled by changing the discharge current in the specified range. In particular, the change in the mass flow rate of the electrolyte is directly related to the change in current. The nature of this dependence was changed by applying an additional plasma-forming gas to the flow. In this case, the condition of the minimum dynamic perturbation of the electrolyte surface was met. To simplify the design and reduce operating costs, air was supplied to the discharge area.

The use of the flowing electrolyte leads to unavoidable heat losses. Part of the heat released in the discharge is carried away by the flow of the electrolyte. It was 
experimentally determined that the heat loss at the liquid cathode was $10 \%$. To ensure such low heat losses, the optimal thickness of the electrolyte layer and its recirculation mode are determined. The speed of movement of the electrolyte through the discharge zone is selected so that it has a temperature of $70 \div 80{ }^{\circ} \mathrm{C}$. The increase in specific electrical conductivity by more than two times led to a decrease in the Joule heat release inside the electrolyte. The heat loss at the anode varied slightly and amounted to $\sim 20 \%$. The total thermal efficiency of the plasma generator is in the range of $0.65 \div 0.75$.

The average mass temperature of the plasma flow in the studied current range reached $2000^{\circ} \mathrm{C}$ (Figure 3). This value is slightly higher than the limit value that can be fixed by a platinum-rhodium thermocouple PR 30/6. The measuring probe was moved using a threecoordinate device capable of moving the probe in three mutually perpendicular directions.

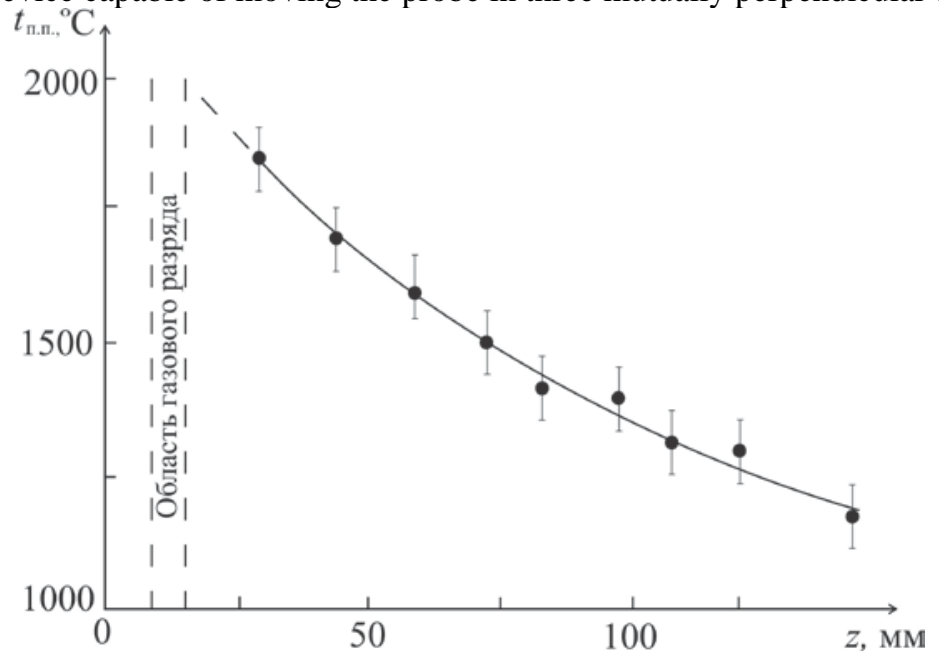

Fig. 3. Plasma flow temperature. Anode - plate. $l_{0}=2 \mathrm{~mm}$. Electrolyte - water solution $\mathrm{Na}_{2} \mathrm{SO}_{4}$.

The current density is the determining factor for the temperature value in the discharge region. Given that the discharge must have a diffuse structure, the limit values of this parameter at the cathode are set from 0.8 to $1.0 \mathrm{~A} / \mathrm{cm}^{2}$. The current density at the anode was 1.5 times higher. This range of values corresponds to the optimal operating mode of the plasma generator for processing carbon-containing waste.

\section{Conclusions}

The parameters of the plasma flow obtained by means of a vapor-gas discharge with a liquid cathode are close to the parameters of the flows created by steam-water electric arc plasma torches. The plasma generator developed and investigated in this work is used for gasification of solid household waste containing hydrocarbon compounds.

\section{References}

1. Altovsky G.S., Bernadiner M.N., Ivanov V.V. Prospects for high-temperature steam gasification of waste using plasma energy sources. // EKIP. - 2011. - № 2. - P. 8-11.

2. Gudim Yu.A., Golubev A.A. Waste-free technology for high-temperature disposal of unsorted household solid waste. // EKIP. - 2009. - № 2. - P. 4-7.

3. Artyomov A.V., Pereslatsev A.V., Krutyakov Yu.A. et al. Environmental aspects of plasma solid waste recycling. // EKIP. - 2011. - № 9. - P. 20-23. 
4. Artyomov A.V., Pereslatsev A.V., Krutyakov Yu.A. et al. Plasma technologies for processing hydrocarbon raw materials and waste. // EKIP. - 2011. - №10. - P. 18-23.

5. Tazmeev, K.K., Tazmeev, B.K. Low-current electric arc in the open air between the end of the cathode and long vertical anode. // 2015. Journal of Physics: Conference Series. 652(1), 012038.

6. Tazmeev G.K., Timerkaev B.A., Tazmeev K.K., Arslanov I.M., Tazmeev B.K., Sarvarov F.S. Study of gas discharge with a liquid cathode at maximum thermal load to the cathode // Journal of Physics: Conference Series. 2017. 789(1), 012060.

7. Tazmeev, K.K., Arslanov, I.M., Tazmeev, B.K., Tazmeev, G.K. Formation of powerful plasma flow from substance of liquid electrolyte cathode. // Journal of Physics: Conference Series. 2019. 1393(1), 012061. 